all ages and affects both sexes equally. The effect on vision can be devastating due to retinal arteritis, retinal necrosis and rhegmatogenous retinal detachment. It is most commonly caused by reactivation of latent infection by varicella zoster virus or HSV but has also been described occurring during herpes simplex encephalitis, soon after shingles and within a month of the onset of chickenpox. Most patients are healthy with no significant medical history although immunosuppressed patients following renal transplantation and acquired immune deficiency syndrome patients have been affected, ${ }^{4}$ making HIV testing relevant when risk factors are identified by history or examination.

A definitive approach to management of acute retinal necrosis has yet to be established. The regime in this relatively mild case proved adequate but in more complicated or less responsive cases, treatment with systemic steroids and a more prolonged course of acyclovir may be appropriate. In any event,

1 Whitley RJ, Alford CA, Hirsch MS, et al. Vidarabine versus acyclovir therapy in herpes simplex encephalitis. $N$ Engl $\mathcal{F}$ Med 1986; 314: 144-9.

2 Gartry DS, Spalton DJ, Tilzey A, Hykin PG. Acute retinal necrosis syndrome. $B r f$ Ophthalmol 1991; 75: 292-7.

3 Sekizawa T, Hara S, Kiyosawa M, Openshaw H, Kogure K. Acute retinitis 2 years after recovery from herpes simplex encephalitis. Neurology 1991; 41: 456. this and other reports of relapse following herpes simplex encephalitis raise doubts about the adequacy of the conventional 10-day regime of acyclovir for eradication of HSV. The recent availability of polymerase chain reaction for detection of HSV DNA has resulted in the recommendation that it should now be the standard for the diagnosis of herpes simplex encephalitis; it also provides the prospect of establishing more accurately the appropriate duration and adequacy of anti-viral therapy in each individual case. A controlled trial to determine if prolonged acyclovir therapy based on the presence of HSV DNA in the CSF can prevent relapse is underway. ${ }^{5}$

\section{Final diagnosis}

Acute retinal necrosis occurring as a late complication of herpes simplex encephalitis.

Keywords: retinal necrosis, herpes simplex encephalitis, acyclovir, polymerase chain reaction

4 Friedman SM, Mames RN, Sleasman JW, Whitcup SM Acute retinal necrosis after chickenpox in a patient with acquired immune deficiency syndrome. Arch Ophthalmol 1993; 111: $1607-8$

5 Kaneko K, Watanabe H, Hikita M, Tokita A, Yabuta K Cessation of antiviral therapy for herpes simplex encephalitis. F Pediatr 1994; 124: 490

\title{
An unusual cause of large bowel obstruction
}

\author{
Faisal Mihaimeed, Audrey Yong, Trevor Paes
}

A 42-year-old woman was re-admitted 10 days following total abdominal hysterectomy for fibroids with abdominal pain and vomiting. She had undergone Nissen's fundoplication for hiatus hernia via a left thoracotomy six months previously. An initial diagnosis of large bowel obstruction was made following routine investigations, which revealed distended loops of small and large bowel with normal chest X-ray. She was initially managed with intravenous fluids and nasogastric suction. Fifteen hours later she developed severe left-sided chest pain with shortness of breath. A repeat chest X-ray was performed (figure).

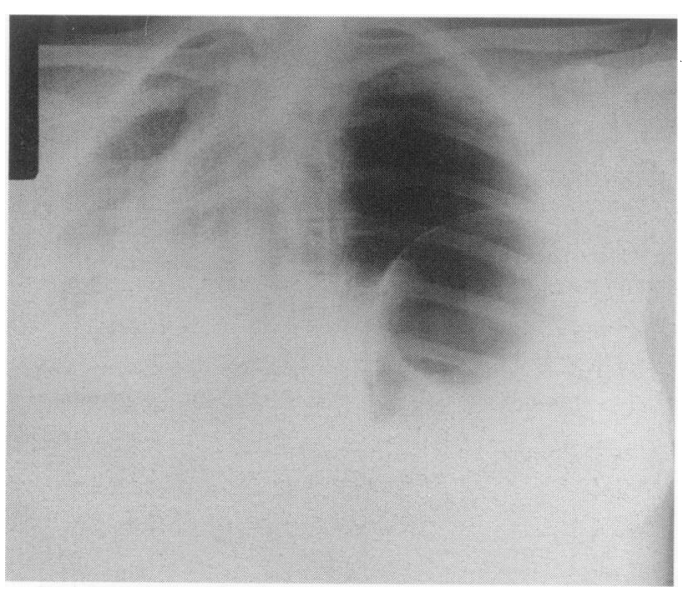

Figure Chest X-ray

\section{Here Chest X-ray}

\section{Questions}

1 What is the diagnosis?

2 Describe the immediate management of this patient
Department of

General Surgery, The

Hillingdon Hospital

Trust,

Pield Heath Road,

Uxbridge UB8 3NN,

UK

F Mihaimeed

A Yong

T Paes

Correspondence to $\mathrm{Dr} A$ Yong

Accepted 7 March 1996 


\section{Answers}

QUESTION 1

There is a left-sided pneumothorax with an obvious loop of bowel above the left hemidiaphragm. In view of the previous history of Nissen's fundoplication, the most likely diagnosis is of a perforated loop of colon in a diaphragmatic hernia causing a secondary tension pneumothorax.

\section{QUESTION 2}

Tension pneumothorax is a life-threatening condition that requires immediate treatment with the insertion of a chest drain. In view of the presence of bowel in the left hemithorax, the chest drain was placed in the second intercostal space. Feculent fluid in the chest drain confirmed the diagnosis. Following adequate resuscitation and intravenous antibiotics, urgent exploration and repair of the defect needs to be undertaken. This was performed via the left thoraco-abdominal approach. At operation, a posterolateral strangulated diaphragmatic hernia of distal transverse colon was found. The portion of gangrenous bowel was excised with subsequent primary end-to-end anastomosis and defunctioning proximal colostomy. The diaphragmatic defect was closed with non-absorbable mattress sutures. Meticulous lavage with saline was performed and a further basal chest drain inserted. Post-operatively, she developed adult respiratory distress syndrome. This was successfully treated on the intensive care unit and she was discharged after a total stay of three weeks.

\section{Discussion}

Diaphragmatic herniae may be congenital or acquired. The latter usually arise in association with multiple trauma or previous thoracoabdominal surgery. A wide range of complications of diaphragmatic herniae have been reported. ${ }^{2,3}$ These include herniation of the stomach, small or large bowel, gall bladder, liver or spleen. Presentation may be immediate or delayed. In the trauma setting, symptoms and signs may be masked by concurrent

1 Beal SL, Mackennan M. Blunt diaphragm rupture, morbid injury. Arch Surg 1988; 123: 828.

2 Hood RM. Traumatic diaphragmatic hernia (review). Ann Thorac Surg 1971; 12: 311.

3 Symbas PN, Vallis SE, Hatcher CR Jr. Blunt and penetrating diaphragmatic injuries with or without herniation of organs into the chest. Ann Thorac Surg 1986; 42: 158.

\section{Learning points}

- acquired diaphragmatic hernia should be suspected in any patients who present with acute abdomen and a past history of chest injury or thoraco-abdominal surgery

- chest X-ray and its correct interpretation is of paramount importance when making an early diagnosis

- once diagnosed, all diaphragmatic herniae should be repaired without delay

injuries. Similarly, congenital diaphragmatic herniae may present during the immediate postnatal period, during childhood, or occasionally in adult life. The clinical presentation includes symptoms and signs of respiratory distress, cardiac disturbance, and/or bowel obstruction.

A chest X-ray showing bowel loops above the hemidiaphragm is diagnostic of diaphragmatic hernia. However this finding is not always present, ${ }^{4}$ and there should be a high index of clinical suspicion if the chest X-ray shows an abnormal diaphragmatic contour and there is a past history of thoraco-abdominal injury or surgery, especially in the presence of mild dilatation of the colon and abdominal pain. Contrast studies may be helpful in establishing the diagnosis.

The mortality from diaphragmatic hernia varies greatly, dependent on the general condition of the patient and the nature of the herniation. When repaired electively, the mortality rate should approach zero. However, in the presence of strangulated or gangrenous bowel, the mortality rate has been quoted as being as high as $80 \% .^{5}$ Surgical toilet, lavage and chest drainage remain the mainstay of treatment, along with intravenous antibiotics and ventilatory support.

\section{Final diagnosis}

Diaphragmatic incisional hernia with tension pneumothorax.

Keywords: diaphragmatic hernia, tension pneumothorax

4 Cruz CJ, Minnagi H. Large bowel obstruction resultin from traumatic diaphragmatic hernia. Imaging findings in four cases. AfR 1994; 162: 843-5.

5 Hegarty MM. Delayed presentation of traumatic diaphragmatic hernia. Ann Surg 1978; 188: 229. 